\title{
Model predictive control design for multivariable processes in the presence of valve stiction ${ }^{\star}$
}

\author{
Riccardo Bacci di Capaci ${ }^{\mathrm{a}, \mathrm{b}}$, Marco Vaccari ${ }^{\mathrm{a}}$, Gabriele Pannocchia ${ }^{\mathrm{a}}$ \\ ${ }^{a}$ Department of Civil and Industrial Engineering, University of Pisa, Pisa, Italy \\ ${ }^{b}$ e-mail: riccardo.bacci@ing.unipi.it
}

\begin{abstract}
This paper presents different formulations of Model Predictive Control (MPC) to handle static friction in control valves for industrial processes. A fully unaware formulation, a stiction embedding structure, and a stiction inversion controller are considered. These controllers are applied to multivariable systems, with linear and nonlinear process dynamics. A semiphysical model is used for valve stiction dynamics and the corresponding inverse model is derived and used within the stiction inversion controller. The twomove stiction compensation method is revised and used as warm-start to build a feasible trajectory for the MPC optimal control problem. Some appropriate choices of objective functions and constraints are used with the aim of improving performance in set-points tracking. The different MPC formulations are reviewed, compared, and tested on several simulation examples. Stiction embedding MPC proves to guarantee good performance in set-points tracking and also stiction compensation, at the expense of a lower robustness with respect to other two formulations.
\end{abstract}

Keywords: Model predictive control, control valves, static friction, stiction modeling and compensation.

\section{Introduction}

Control valves are the most commonly used actuators in the process industries. Unfortunately, in many cases valves not only contain static nonlinearity (e.g. saturation), but also dynamic nonlinearity including backlash, friction, and hysteresis. Deadband due to backlash and mostly static friction (stiction) is a typical root source of the valve problems. A control valve with excessive deadband may not even respond to small changes in control action. As a result, these malfunctions may produce sustained oscillations in process variables, decrease the life of control valves, and generally, lead to inferior quality endproducts causing reduced profitability of the whole industrial plant [2]. Hence, it appears that the potential benefits of using advanced control algorithms, as model predictive control (MPC), could be diminished because of poor valves, especially if their faults and malfunctions are not expressly considered in the plant model.

As a matter of fact, MPC has been used as an useful tool to improve control performance in the presence of various types of actuator faults, thus forming effective examples of fault tolerant control (FTC), as in [3] and [4]. In addition, MPC has been specifically applied as a compensation strategy for several types of control valve malfunctions. In particular, the first MPC-based formulation was developed in [5], using a mixedinteger quadratic programming (MIQP) on constraints of the input. An inverse backlash model and valve saturation are incorporated in the controller to overcome the deadband associated with backlash. Later, this structure has been applied to a

${ }^{\star} \mathrm{A}$ preliminary version of this paper has been presented in [1]. system with valve stiction in [6]. Due to the high computational burden and the resulting feedback effect, this approach may be inefficient in the case of severely nonlinear systems (high stiction) or highly dimensional systems. Further investigations of the same method in the case of valve stiction within the process, but not in the model, have been presented in [7].

Rodriguez and Heath [8] have proposed a formulation which reduces the bounds of optimization variables computed by the MPC, by trying to delete different types of valve nonlinearity, and by reducing the problem to a purely linear structure. The controller is indeed in series with a block that applies the inverse model for deadzone, backlash or stiction to the MPC output and sends this signal to the faulty valve, which can eventually saturate. Recently, Durand and Christofides [9] have presented an economic MPC structure which includes a detailed physical stiction model, constraints on the magnitude and rate of change of the input, and is combined with a slave controller of PI-type that regulates the valve output to its MPC set-point. This approach comprises a compensation strategy for nonlinear process systems, which can prevent the MPC from requesting physically unrealistic control actions due to stiction. Later, the same authors have replaced in [10] the first-principles model for the valve layer with a procedure for developing empirical models based only on data of valve set-point and flow rate. This approach incorporates a logic structure that activates different equations depending on the valve condition (that is, sticking or sliding phase): this forms a piecewise model where set-point changes may set which equations have to be chosen. The empirical model proves to be less stiff than the first-principles model and may improve the computation time with limited violations of process constraints. 

the MPC output, while $w$ and $v$ are white Gaussian noise. In [1]

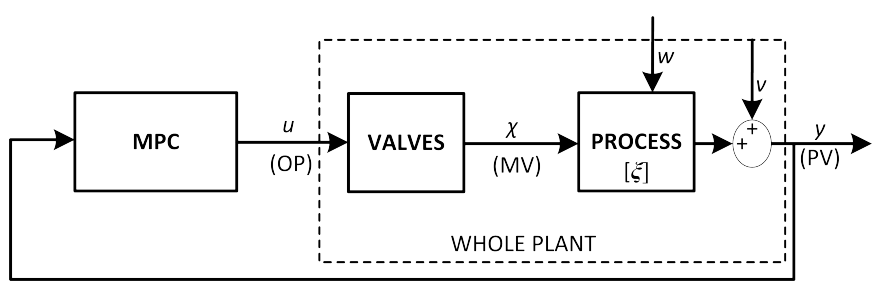

Figure 1: The closed-loop system with (sticky) control valves followed by the process.

As stated before, when stiction is present, the valve is not effective in following the command signal imposed by the controller. As a result, a limit cycle with sustained oscillations is typically produced in the proximity of the steady-state operating points. One way of reducing stiction effects is to explicitly take this malfunction into account in MPC design so that an mproved performance could be obtained. As in many other foult torant control systems, where the fault estimate is crucial, for a good stiction tolerant MPC, a solid estimate of the stiction amount is needed, and the sticky valve must be properly located, especially when the system is multidimensional. For this purpose, well-established techniques of oscillation detection [11], and stiction diagnosis and quantification [12] could be used and adapted as necessary.

This paper is focused on designing and comparing different strategies of model predictive controller to handle static friction in control valve. Among three main different solutions, one MPC formulation considers valve stiction explicitly, using a semiphysical model [13] which is proved to give very close responses with respect to well-established first-principles models. The objective of this model-based approach is to compensate for the undesired effects of stiction on the controlled systems. Note that no method for valve stiction quantification has been expressly used or derived. Conversely, being stiction quantifisumed as prior knowledge for predictive controllers.

The various controllers have been previously derived for single-input single-output (SISO) systems with linear process dynamics, as the nonlinearity came only from the valve [1]. In this work, the formulations have been refined and extended to multidimensional processes and nonlinear (and linearized) systems. An appropriate input sequence, derived from the twomove stiction compensation method and used as warm-start for MPC, is developed to improve set-point tracking performance. The considered MPC formulations are analyzed and compared using as test bench several simulation examples.

\section{Problem definition}

The whole multivariable plant is formed by the control valves followed by the process dynamics as shown in Figure 1. In detail, $\chi$ and $y$ are the process input and output, that is, the valves output and the control variables, respectively; then, $u$ is

the case of SISO system was studied. The system comprised a nonlinearity with memory for the valve followed by a linear dynamics for the process, thus forming an extended Hammerstein structure for the whole plant. In this work, applications to MIMO systems with linear and nonlinear processes are presented.

In particular, the process dynamics is as follows:

$$
\begin{aligned}
\xi_{k+1} & =f_{P}\left(\xi_{k}, \chi_{k}\right)+w_{k} \\
y_{k} & =h_{P}\left(\xi_{k}\right)+v_{k}
\end{aligned}
$$

where variables are $\chi \in \mathbb{R}^{m}, u \in \mathbb{R}^{m}, y \in \mathbb{R}^{p}$, and $\xi \in \mathbb{R}^{n}$ (the process states), being $n$ the model dimension; while functions are $f_{P}: \mathbb{R}^{n} \times \mathbb{R}^{m} \rightarrow \mathbb{R}^{n}, h_{P}: \mathbb{R}^{n} \rightarrow \mathbb{R}^{p}$. Whereas, the dynamics of the $m$ valves is described by a data-driven stiction model:

$$
\chi_{k}=\varphi\left(\chi_{k-1}, u_{k}\right)
$$

expressed by the nonlinear function $\varphi: \mathbb{R}^{m} \times \mathbb{R}^{m} \rightarrow \mathbb{R}^{m}$, which is later discussed. Overall, the output of valve system $\chi$ represents the first $m$ components of the state vector of the complete plant: $z_{k}=\left[\chi_{k-1}^{T}, \xi_{k}^{T}\right]^{T}$, so that $z \in \mathbb{R}^{n_{z}}$, being $n_{z}=m+n$. Therefore, the whole dynamics can be written as:

$$
\begin{aligned}
z_{k+1}=\left[\begin{array}{c}
\chi_{k} \\
\xi_{k+1}
\end{array}\right]=\phi_{P}\left(z_{k}, u_{k}\right) & =\left[\begin{array}{c}
\varphi\left(\chi_{k-1}, u_{k}\right) \\
f_{P}\left(\xi_{k}, \varphi\left(\chi_{k-1}, u_{k}\right)\right)+w_{k}
\end{array}\right] \\
y_{k} & =\zeta_{P}\left(z_{k}\right)+v_{k}
\end{aligned}
$$

where $\phi_{P}: \mathbb{R}^{n_{z}} \times \mathbb{R}^{m} \rightarrow \mathbb{R}^{n_{z}}$, and $\zeta_{P}: \mathbb{R}^{n_{z}} \rightarrow \mathbb{R}^{p}$, being $\zeta_{P}\left(z_{k}\right)=$ $h_{P}\left(\xi_{k}\right)$. Note that in the present discussion, all actuators are assumed to be control valves, possibly affected by static friction. If some actuators are not valves, suitable simplifications can be easily made.

\subsection{Proposed MPC approaches}

Three different MPC approaches are presented and compared in this work. The first formulation is a "stiction unaware MPC", with a partial nonlinear formulation since it completely disregards the valves dynamics and uses only the nonlinear process model for the whole plant (see Figure 2). Secondly, a "stiction embedding MPC" is considered, as shown in Figure 3 This controller is aware of the stiction presence, as it uses an extended model - comprised of valves and process dynamics thus forming a full nonlinear formulation.

Finally, the third approach is also aware of stiction, but it has an explicit model for the inverse dynamics of stiction $\left(\tilde{\varphi}^{-1}\right)$, as in Figure 4. In this case, $\tilde{u}$ is the MPC output, subject to optimization, which forms input to stiction inverse model, and $u=\tilde{\varphi}^{-1}(\tilde{u})$ is the output of the whole controller. Note that, for a perfect stiction inversion, one get $\varphi\left(\tilde{\varphi}^{-1}(\tilde{u})\right)=\tilde{u}$, and then $\tilde{u} \equiv \chi$. This type of formulation, introduced by [8], has the advantage of considering expressly stiction dynamics, but it is mainly beneficial when the controller uses a linear model, that is, it is based on a linearized process dynamics.

\subsection{Valve stiction modeling}

For a healthy linear control valve, input $(u)$ and output $(\chi)$ signals are equal (or at least proportional) at all times. But in the case of stiction, the valve acts like a nonlinear element and 


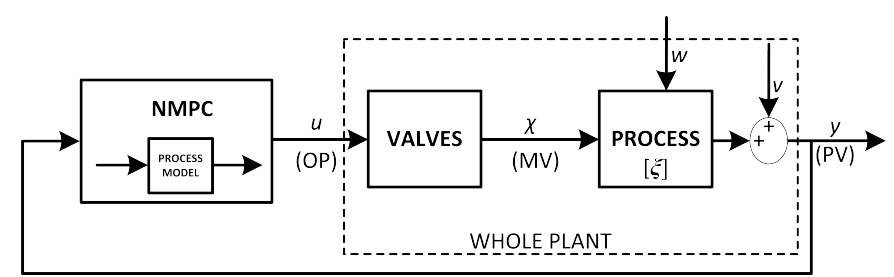

Figure 2: Closed-loop system with "stiction unaware MPC".

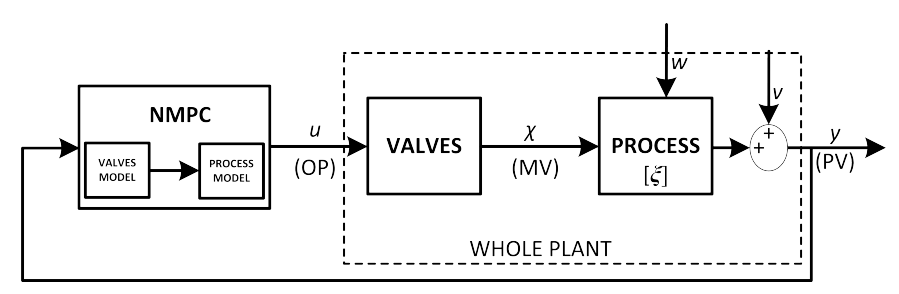

Figure 3: Closed-loop system with "stiction embedding MPC".

these two signals clearly differ. Note that stiction has been historically observed and studied in pneumatic globe valves with sliding stem and spring-diaphragm actuation system [2]. However, similar sticky behaviors can also occur in rotary pneumatic valves and even in electric control valves. Pneumatic and electric control valves differ actually only for the actuator, while the body and the plug, subjected to the majority of friction forces, can be exactly the same [14].

Stiction in control valves can be described both by detailed physical models, then by purely empirical models [15]. Physical models use Newton's second law of motion and classical forces balance on the valve. However, there are two main disadvantages of these first-principles models. Firstly, several physical parameters, also related to the valve's size, which are actually difficult to estimate, must be known. Secondly, computational times may be excessively long for practical purposes because cumbersome numerical integrations are necessary. Therefore, physical models are not often used in industrial applications.

On the other hand, data-driven (empirical) modeling approaches can get over the previous two drawbacks, by limiting the number of parameters and the computational burden. However, such models may also present some disadvantages. In fact, they cannot fully capture the dynamics of the valve, since, for example, not all the proposed models passed the specific openloop tests applied by following the standards of International Society of Automation (ISA) [15].

When a fast response from the control valve is assumed, the transient behavior can be ignored, and a static - but with memory - nonlinear function can be used to approximate the valve's dynamic response, that is, only steady-state values of stem position are considered (see Figure 5). The standard empirical [16] or the semiphysical model [13] by He and coworkers are thus suitable to reproduce the valve response generated by physical stiction models without involving computationally intensive numerical integration.

He's semiphysical stiction model. In this paper, we choose to use He's semiphysical model [13], which includes stiction in

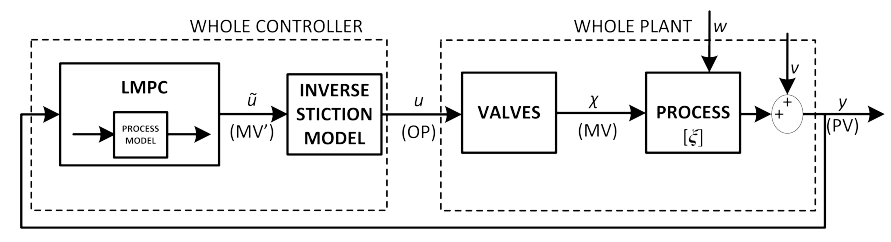

Figure 4: Closed-loop system with "stiction inversion MPC".

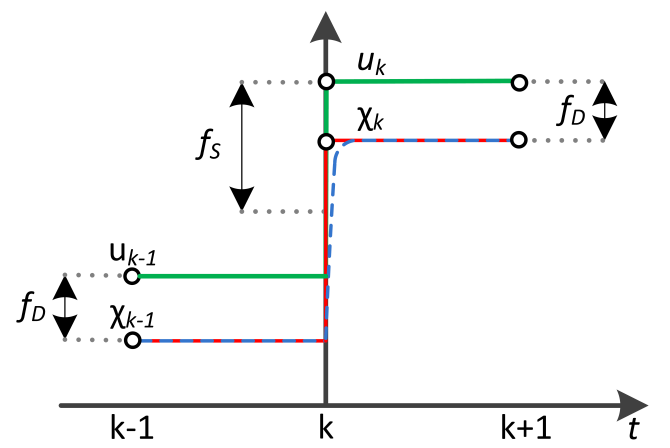

Figure 5: Steady-state approximation for valve stiction dynamics.

every valve movement, and reproduces accurately the valve signature obtained with the physical model in the case of low values of viscous friction $F_{v}$ [13]. As said, preliminary version of this study has been carried out in [1] using He's standard model.

The flowchart of He's semiphysical model is given in Figure 6 The generic $i$-th sticky valve has nonlinear dynamics with memory $\chi_{k}^{(i)}=\varphi\left(\chi_{k-1}^{(i)}, u_{k}^{(i)}\right)$, expressed by the following two relations:

$$
\chi_{k}^{(i)}=\left\{\begin{array}{lll}
\chi_{k-1}^{(i)}+M \cdot\left[e_{k}^{(i)}-\operatorname{sign}\left(e_{k}^{(i)}\right) f_{D}^{(i)}\right] & \text { if } & \left|e_{k}^{(i)}\right|>f_{S}^{(i)} \\
\chi_{k-1}^{(i)} & \text { if } & \left|e_{k}^{(i)}\right| \leq f_{S}^{(i)}
\end{array}\right.
$$

for $i=1, \ldots, m$, being $m$ the number of valves, where $f_{S}^{(i)}$ and $f_{D}^{(i)}$ are static and dynamic friction parameters, respectively, and $e_{k}^{(i)}=u_{k}^{(i)}-\chi_{k-1}^{(i)}$. Note that $e_{k}^{(i)}$ is a sort of valve position error, and $f_{S}^{(i)} \geq f_{D}^{(i)}$ by definition.

The parameter $M$, which accounts for the overshoot observed in the physical model, can be assumed as a constant ( $M=1.99$ ) for different valve physical parameters when $F_{v} \simeq 0$ [13]. Therefore, this three-parameter model reduces to a modified version of the standard two-parameter model of $\mathrm{He}$, and by

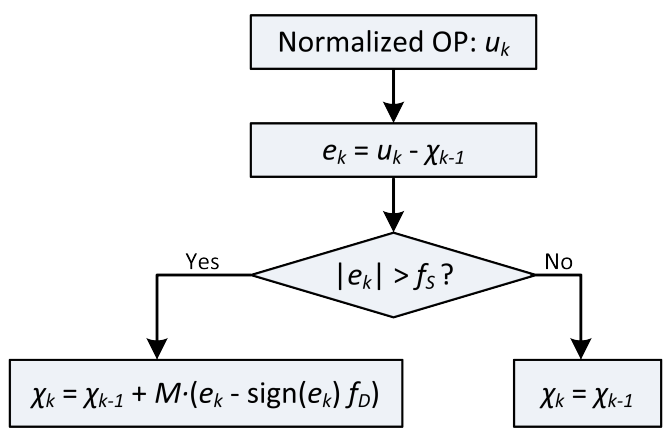

Figure 6: He's semiphysical stiction model [13]. 
Therefore, the stiction nonlinearity $\varphi$ in $(3)$ is formed by a set of three linear and parallel relations for each valve, thus constituting a sort of switching "multi-mode" model to be considered along with the dynamics of the process, to form a discontinuous model. Finally, it has to be noted that the proposed methodology and formulations of MPC could be derived also with other types of data-driven stiction models, as [17, 18].

\section{MPC controller design}

In this section the considered formulations of MPC are detailed, by introducing an empirical stiction inverse model, some specific choices for modules and tuning parameters, and a suitable warm-start based on a stiction compensation method.

\subsection{Stiction inverse model}

The "stiction inverse MPC" formulation presented in Figure 4 requires to invert the stiction nonlinearity to obtain the control sequence, that is, $u=\tilde{\varphi}^{-1}(\tilde{u})$. Starting from He's semiphysical model in (5), by assuming $\tilde{u}=\chi$ and knowing at each sampling $\tilde{u}_{k}$ and $\tilde{u}_{k-1}$, which compose inputs to the stiction inverse model, one can write that:

- if $\tilde{u}_{k}^{(i)} \neq \tilde{u}_{k-1}^{(i)}$ then:

$$
u_{k}^{(i)}= \begin{cases}\frac{1}{M}\left[\tilde{u}_{k}^{(i)}+M f_{D}^{(i)}-\tilde{u}_{k-1}^{(i)}(1-M)\right] & \Leftrightarrow u_{k}^{(i)}-\tilde{u}_{k-1}^{(i)}>f_{S}^{(i)} \\ \frac{1}{M}\left[\tilde{u}_{k}^{(i)}-M f_{D}^{(i)}-\tilde{u}_{k-1}^{(i)}(1-M)\right] & \Leftrightarrow u_{k}^{(i)}-\tilde{u}_{k-1}^{(i)}<-f_{S}^{(i)}\end{cases}
$$

- if $\tilde{u}_{k}^{(i)}=\tilde{u}_{k-1}^{(i)}$ then $u_{k}^{(i)} \in\left[\tilde{u}_{k-1}^{(i)}-f_{S}^{(i)}, \tilde{u}_{k-1}^{(i)}+f_{S}^{(i)}\right]$

Then, by substituting the expression of $u_{k}^{(i)}$ in the two inequalities, one gets:

$$
u_{k}^{(i)} \begin{cases}=\tilde{U}_{k}^{(i)}+f_{D}^{(i)} & \text { if } \tilde{u}_{k}^{(i)}-\tilde{u}_{k-1}^{(i)}>M\left(f_{S}^{(i)}-f_{D}^{(i)}\right) \\ =\tilde{U}_{k}^{(i)}-f_{D}^{(i)} & \text { if } \tilde{u}_{k}^{(i)}-\tilde{u}_{k-1}^{(i)}<M\left(f_{D}^{(i)}-f_{S}^{(i)}\right) \\ \in\left[\tilde{u}_{k-1}^{(i)}-f_{S}^{(i)}, \tilde{u}_{k-1}^{(i)}+f_{S}\right] & \text { if } \tilde{u}_{k}^{(i)}-\tilde{u}_{k-1}^{(i)}=0 \\ \text { is undefined } & \text { otherwise }\end{cases}
$$

where $\tilde{U}_{k}^{(i)}=\frac{1}{M}\left[\tilde{u}_{k}^{(i)}-\tilde{u}_{k-1}^{(i)}(1-M)\right]$. Figure 7 shows a schematic representation of the function $\tilde{\varphi}^{-1}$ for the $i$-th valve. This stiction inverse model has an incomplete domain in $\mathbb{R}$, it admits unique values for $\tilde{u}_{k}-\tilde{u}_{k-1}>M\left(f_{S}-f_{D}\right) \geq 0$ and for $\tilde{u}_{k}-\tilde{u}_{k-1}<M\left(f_{D}-f_{S}\right) \leq 0$, it is multivalued for $\tilde{u}_{k}-\tilde{u}_{k-1}=0$, while otherwise is not defined. Note that, to implement this

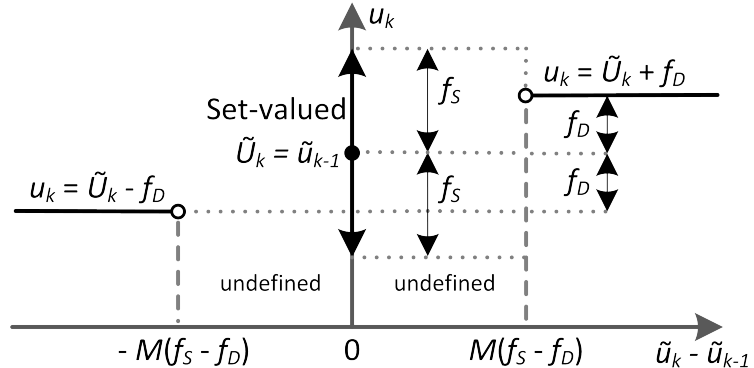

Figure 7: Inverse function $\tilde{\varphi}^{-1}$ for He's semiphysical stiction model.

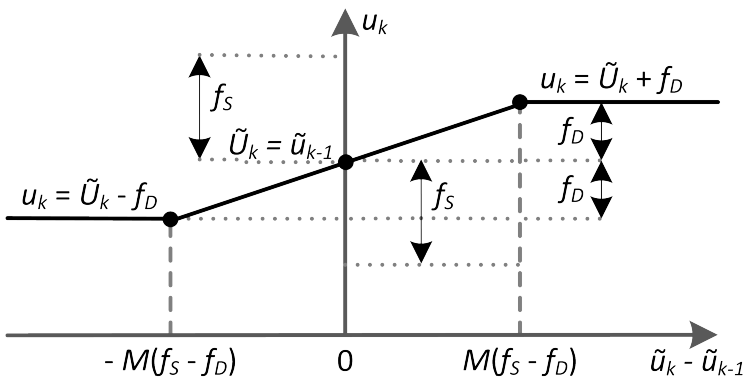

Figure 8: Approximated inverse function $\hat{\varphi}^{-1}$ for He's semiphysical stiction model.

exact model of stiction inverse in the MPC formulation, one should theoretically impose the following nonconnected domain $\tilde{U}$ for values of $\tilde{u}_{k}$ :

$$
\begin{array}{r}
\tilde{\mathbb{U}}=\left\{\tilde{u}_{k}: \tilde{u}_{k}^{(i)}>\tilde{u}_{k-1}^{(i)}+M\left(f_{S}^{(i)}-f_{D}^{(i)}\right) \cup\right. \\
\tilde{u}_{k}^{(i)}<\tilde{u}_{k-1}^{(i)}-M\left(f_{S}^{(i)}-f_{D}^{(i)}\right) \cup \\
\left.\tilde{u}_{k}^{(i)}=\tilde{u}_{k-1}^{(i)}, \forall i=1, \ldots, m\right\}
\end{array}
$$

which is not actually implementable, since it implies a nonconnected set of constraints on $\tilde{u}_{k}$, apart from the special case of pure deadband, that is, $f_{S}^{(i)}=f_{D}^{(i)} \forall i$. Therefore, an approximated inverse model $\left(\hat{\varphi}^{-1} \approx \tilde{\varphi}^{-1}\right)$ is needed to implement a standard MPC. A possible simple solution is to turn the model into a continuous function with linear junctions, as the following:

$u_{k}^{(i)}=\left\{\begin{array}{llll}\tilde{U}_{k}^{(i)}+f_{D}^{(i)} & \text { if } & \tilde{u}_{k}^{(i)}-\tilde{u}_{k-1}^{(i)}>M\left(f_{S}^{(i)}-f_{D}^{(i)}\right) \\ \tilde{U}_{k}^{(i)}-f_{D}^{(i)} & \text { if } & \tilde{u}_{k}^{(i)}-\tilde{u}_{k-1}^{(i)}<-M\left(f_{S}^{(i)}-f_{D}^{(i)}\right) \\ \tilde{U}_{k}^{(i)}+\frac{f_{D}^{(i)}}{M\left(f_{S}^{(i)}-f_{D}^{(i)}\right)}\left(\tilde{u}_{k}^{(i)}-\tilde{u}_{k-1}^{(i)}\right) & \text { if } & \left|\tilde{u}_{k}^{(i)}-\tilde{u}_{k-1}^{(i)}\right| \leq M\left(f_{S}^{(i)}-f_{D}^{(i)}\right)\end{array}\right.$

Figure 8 shows a schematic representation of this approximated 213 stiction inverse for $i$-th valve. Note that for $f_{S}^{(i)}=f_{D}^{(i)}$ the third ${ }_{214}$ condition, that is, when $\tilde{u}_{k}^{(i)}-\tilde{u}_{k-1}^{(i)}=0$, has to be reduced to ${ }_{215}$ $u_{k}^{(i)}=\tilde{U}_{k}^{(i)}$.

Extensive simulations have verified that approximated model (8) equals the exact one (6), that is, $\hat{\varphi}^{-1} \equiv \tilde{\varphi}^{-1}$, only when the difference $\tilde{u}_{k}^{(i)}-\tilde{u}_{k-1}^{(i)}$ is always within the domain of the exact inverse, and then one gets $\tilde{u}^{(i)} \equiv \chi^{(i)}$. Otherwise, the stiction inverse MPC formulation has a structural mismatch and its per- 

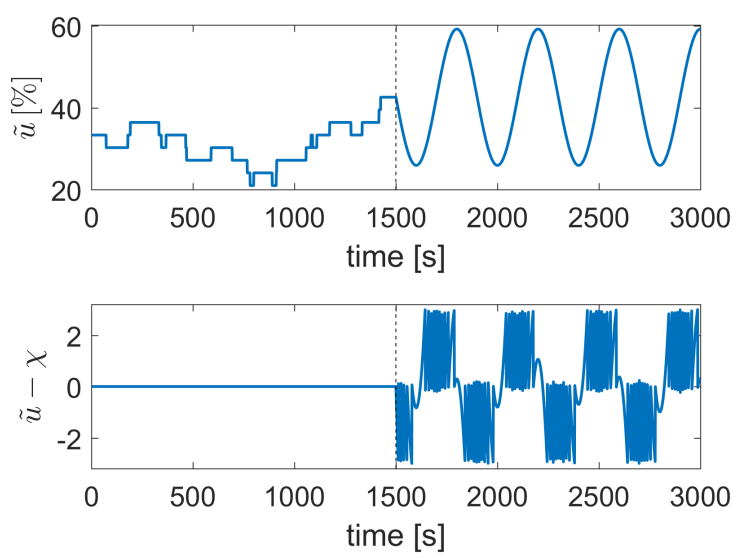

Figure 9: Behavior of stiction inverse model for a test signal $\left(f_{S}=3, f_{D}=1.5\right)$.

For all three formulations an input disturbance model is used. The stiction embedding MPC uses a full nonlinear structure, so that the augmented plant model becomes:

$$
\begin{aligned}
\hat{z}_{k+1 \mid k}=\phi\left(\hat{z}_{k \mid k}, u_{k}, \hat{d}_{k \mid k}\right) & =\left[\begin{array}{c}
\varphi\left(\hat{\chi}_{k-1}, u_{k}\right) \\
f\left(\hat{\xi}_{k \mid k}, \varphi\left(\hat{\chi}_{k-1}, u_{k}\right)+\hat{d}_{k \mid k}\right)
\end{array}\right] \\
\hat{d}_{k+1 \mid k} & =\hat{d}_{k \mid k} \\
\hat{y}_{k} & =\zeta\left(\hat{z}_{k \mid k-1}\right)
\end{aligned}
$$

where $\hat{d} \in \mathbb{R}^{n_{d}}$ is the estimate of input disturbance, where $n_{d}=$ $m$. The stiction unaware MPC uses an incomplete nonlinear structure, since stiction nonlinearity is unmodeled $(z \rightarrow \xi, \phi \rightarrow$ $f, \zeta \rightarrow h)$

$$
\begin{aligned}
\hat{\xi}_{k+1 \mid k} & =f\left(\hat{\xi}_{k \mid k}, u_{k}+\hat{d}_{k \mid k}\right) \\
\hat{d}_{k+1 \mid k} & =\hat{d}_{k \mid k} \\
\hat{y}_{k} & =h\left(\hat{\xi}_{k \mid k-1}\right)
\end{aligned}
$$

For stiction inversion MPC, a linearized model is used, with $z \rightarrow \xi, \phi \rightarrow f$ and $\zeta \rightarrow h$, so that:

$$
\begin{aligned}
\hat{\xi}_{k+1 \mid k} & =A \hat{\xi}_{k \mid k}+B u_{k}+B_{d} \hat{d}_{k \mid k} \\
\hat{d}_{k+1 \mid k} & =\hat{d}_{k \mid k} \\
\hat{y}_{k} & =C \hat{\xi}_{k \mid k-1}
\end{aligned}
$$

being $A=D_{\hat{\xi}} f(\hat{\xi}) \in \mathbb{R}^{n \times n}$ and $B=D_{u} f(u) \in \mathbb{R}^{n \times m}$ the Ja- ${ }^{233}$ cobian matrices of state dynamics with respect to states $\xi$ and input $u$, respectively; $C=D_{\hat{\xi}} h(\hat{\xi}) \in \mathbb{R}^{p \times n}$ is the Jacobian matrix of output dynamics with respect to states $\xi$, and $B_{d} \in \mathbb{R}^{n \times n_{d}}$ is the state disturbance matrix. The standard linear input disturbance model is used: $B_{d}=B$.

The three modules (Estimator, Steady-State Optimizer, Dynamic Optimizer) implemented in the proposed MPC formulations are briefly described below. Note that all modules are executed at each sample time given that the disturbance estimate $\hat{d}$ is updated at each time $k$, and this implies that new targets need to be recomputed.

State estimation. The state estimator receives current output measurement $\left(y_{k}\right)$ and predictions of state $\left(\hat{z}_{k \mid k-1}\right)$ and disturbance $\left(\hat{d}_{k \mid k-1}\right)$. The prediction update is made by:

$$
\left[\begin{array}{c}
\hat{z}_{k \mid k} \\
\hat{d}_{k \mid k}
\end{array}\right]=\left[\begin{array}{c}
\hat{z}_{k \mid k-1} \\
\hat{d}_{k \mid k-1}
\end{array}\right]+K_{k} e_{k}
$$

where $e_{k}=y_{k}-\hat{y}_{k}$ is the prediction error, and $K_{k}$ is the observer gain matrix $\in \mathbb{R}^{\left(n_{z}+n_{d}\right) \times p}$. The Extended Kalman filter, a classical dynamic observer, is used. Therefore, at each sample time $k$, the observer gain matrix $K_{k}$ is computed by solving the following equations:

$$
\begin{aligned}
K_{k} & =\left(P_{k \mid k-1} C_{k}^{T}\right)\left(C_{k} P_{k \mid k-1} C_{k}^{T}+R_{k f}\right)^{-1} \\
P_{k} & =\left(I-K_{k}^{T} C_{k}\right)^{T} P_{k \mid k-1} \\
P_{k+1 \mid k} & =A_{k} P_{k} A_{k}^{T}+Q_{k f}
\end{aligned}
$$

where $A_{k}$ and $C_{k}$ are the Jacobian matrices of the augmented model dynamics and of the output map with respect to the augmented state vector $\left[z^{T}, d^{T}\right]^{T} ; R_{k f} \in \mathbb{R}^{p \times p}$ and $Q_{k f} \in$ $\mathbb{R}^{n_{z}+n_{d} \times n_{z}+n_{d}}$ are the measurements noise and process noise covariance matrices, respectively; $P_{0} \in \mathbb{R}^{n_{z}+n_{d} \times n_{z}+n_{d}}$ is the covariance of the state error at the initial time. It is then imposed $R_{k f}=R_{w n}$, that is, estimated noise covariance matrix equals its actual value. 
Steady-state optimization. The steady-state optimizer computes the state $\left(z_{s s}\right)$, input $\left(u_{s s}\right)$, and output $\left(y_{s s}\right)$ targets to follow the desired external set-points $\left(u_{s p}, y_{s p}\right)$ while respecting the imposed constraints. The optimization problem is as follows:

$$
\left(z_{s s}, u_{s s}, y_{s s}\right)=\arg \min _{z, u, y} \ell_{s s}(u, y)
$$

subject to:

$$
\begin{gathered}
z_{\min } \leq z \leq z_{\max } \\
u_{\min } \leq u \leq u_{\max } \\
y_{\min } \leq y \leq y_{\max } \\
c_{s s}\left(z, u, y, \hat{d}_{k \mid k}\right)=0
\end{gathered}
$$

Dynamic optimization. The dynamic optimizer finds optimal trajectory $(\mathbf{z}, \mathbf{u})$ from current state and input to targets and computes $u_{k}=\mathbf{u}_{k}(0)$. The problem is formulated as follows:

$$
\left[\mathbf{z}_{k}, \mathbf{u}_{k}\right]=\arg \min _{\mathbf{z}, \mathbf{u}} \ell_{d y n}(\mathbf{z}, \mathbf{u})=\sum_{i=0}^{N-1} \ell\left(z_{i}, u_{i}\right)+V_{f}\left(z_{N}\right)
$$

subject to:

$$
\begin{gathered}
z_{\min } \leq z_{i} \leq z_{\max } \\
u_{\min } \leq u_{i} \leq u_{\max } \\
\Delta u_{\min } \leq \Delta u_{i} \leq \Delta u_{\max } \\
y_{\min } \leq y_{i} \leq y_{\max } \\
z_{0}=\hat{z}_{k \mid k} \\
c_{e q}\left(z_{i}, z_{i+1}, u_{i}, y_{i}, \hat{d}_{k \mid k}\right)=0
\end{gathered}
$$

where $N$ is the prediction horizon, and $V_{f}\left(z_{N}\right)=\left(z_{N}-\right.$ $\left.z_{s s}\right)^{T} Q_{N}\left(z_{N}-z_{s s}\right)$ is the terminal weight, with $Q_{N} \in \mathbb{R}^{n_{z} \times n_{z}}$. Also this objective function is quadratic:

$$
\begin{array}{rl}
\ell\left(z_{i}, u_{i}\right)=\left(z_{i}-z_{s S}\right)^{T} & Q\left(z_{i}-z_{s s}\right)+ \\
& \left(u_{i}-u_{s s}\right)^{T} R\left(u_{i}-u_{s s}\right)+\Delta u_{i}^{T} S \Delta u_{i}
\end{array}
$$

Controller tuning. Some general details about tuning parameters are given, even though specific numerical values depend on the various case studies. In the case of valve stiction, steadystate matrices can be chosen as $Q_{s s}=I_{p}$ and $R_{s s}=0$, so that deviations from targets of inputs $u_{s s}$ are not weighted at all. This choice is appropriate because valve stiction dynamics (5) admits multiple steady-states; in particular, when the valve is sticking, for a given steady output a range of inputs of width of $2 f_{S}$ is possible, that is, $u_{s s} \in\left[\chi_{s s}-f_{S}, \chi_{s s}+f_{S}\right]$.

For stiction embedding MPC, the state penalty matrix $Q$ can be chosen as a pure diagonal matrix with higher values in the first $m$ elements in order to weigh deviations from steady-state position of valves. For both stiction embedding and stiction inversion MPC formulations, the following constraints on input rate of change are considered: $\Delta u_{\min , \max }=\mp a f_{S}$, where $a>2$.

\subsection{A suitable warm-start for stiction embedding MPC}

In order to get good tracking performance and move variables to their targets by avoiding sustained oscillations induced by valve stiction, a suitable warm-start should be given to the dynamic optimizer of MPC. A general formulation of warmstart can be obtained by solving the following dynamic optimization problem:

$$
\min _{\hat{\chi}_{k}, \hat{\chi}_{k+1}, u_{k+j}}\left(\hat{\chi}_{k-1}-\hat{\chi}_{k}\right)^{2}+\left(\hat{\chi}_{k}-\hat{\chi}_{k+1}\right)^{2}+\left(\hat{\chi}_{k+1}-\hat{\chi}_{s s}\right)^{2}
$$

subject to:

$$
\begin{gathered}
\hat{\chi}_{k}=\varphi\left(\hat{\chi}_{k-1}, u_{k}\right) \\
\hat{\chi}_{k+1}=\varphi\left(\hat{\chi}_{k}, u_{k+1}\right) \\
\hat{\chi}_{s s}=\varphi\left(\hat{\chi}_{k+1}, u_{k+2}\right) \\
\hat{\chi}_{s s}=\varphi\left(\hat{\chi}_{s s}, u_{k+3}\right)
\end{gathered}
$$

where $j=0, . .3$. The problem computes four moves $\left(u_{k}, u_{k+1}, u_{k+2}, u_{k+3}\right)$ by optimizing on $\hat{\chi}_{k}$ and $\hat{\chi}_{k+1}$, and by assuming $\hat{\chi}_{k+3}=\hat{\chi}_{k+2}=\hat{\chi}_{s s}$.

In the proposed formulation (3), the valves output represent the first $m$ components of the state vector of the complete plant model. Therefore, at each sampling time, the steady-state optimization module of stiction embedding MPC (9) can compute a suitable steady-state target $\left(\chi_{s s}\right)$ also for the valves output:

$$
\begin{aligned}
z_{s s}=\phi\left(z_{s s}, u_{s s}\right)=\left[\begin{array}{c}
\chi_{s s} \\
\xi_{s s}
\end{array}\right] & =\left[\begin{array}{c}
\varphi\left(\chi_{s s}, u_{s s}\right) \\
f\left(\xi_{s s}, \varphi\left(\chi_{s s}, u_{s s}\right)\right)
\end{array}\right] \\
y_{s s} & =\zeta\left(z_{s s}\right)=y_{s p}
\end{aligned}
$$

Alternatively, a particular input sequence could be used as firstguess trajectory. This suitable warm-start is inspired by a new version of the two-move stiction compensation method. Introduced by [20], the "two-move compensator" ought to remove oscillations on control variable, and keep the valve output at its steady-state value, by performing at least two moves in opposite directions. Afterwards, further and improved implementations of stiction compensators based on this approach have been then developed in [21], [22], and [23]. 
In this work, the following sequence is given as warm-start to input signal of each $m$ valve on the basis of semiphysical He's stiction model (5):

$$
\begin{gathered}
u_{k}=\left\{\begin{array}{lll}
u_{k-1}+a f_{S} & \text { if } & u_{k-1} \geq \chi_{s s} \\
u_{k-1}-a f_{S} & \text { if } & u_{k-1}<\chi_{s s}
\end{array}\right. \\
u_{k+1}=\left\{\begin{array}{lll}
\chi_{k}+f_{D} & \text { if } & u_{k} \geq \chi_{k} \\
\chi_{k}-f_{D} & \text { if } & u_{k}<\chi_{k}
\end{array}\right. \\
u_{k+2}=\left\{\begin{array}{lll}
X_{k+2}-f_{D} & \text { if } & u_{k+1} \geq \chi_{s s} \\
X_{k+2}+f_{D} & \text { if } & u_{k+1}<\chi_{s s}
\end{array}\right. \\
u_{k+3}=\left\{\begin{array}{lll}
\chi_{k+2}+f_{D} & \text { if } & u_{k+2} \geq \chi_{k+2} \\
\chi_{k+2}-f_{D} & \text { if } & u_{k+2}<\chi_{k+2}
\end{array}\right. \\
u_{k+j}=u_{k+3}\left(=u_{s s}\right) \text { if } \quad j>3
\end{gathered}
$$

where $X_{k+2}=\frac{1}{M}\left[\chi_{s s}-\chi_{k+1}(1-M)\right]$. The first input $u_{k}$ (for $j=0$ ) moves the valve stem away from its stuck position, if $a>2$. By observing (5), it is evident that the maximum value of the difference between valve input and output that does not cause a movement in the valve is $\left|u_{k}-\chi_{k-1}\right|=f_{S}$. If $u_{k-1} \geq \chi_{s s}$, in the worst case $u_{k-1}-\chi_{k-1}=-f_{S}$. Therefore, if $a>2$, one gets $\left|u_{k}-\chi_{k-1}\right|>f_{S}$ and moves the valve: $\chi_{k} \neq \chi_{k-1}$ (see Figure 11). Then, for the second movement $u_{k+1}$ (for $j=1$ ), the input is moved towards the actual valve position $\chi_{k}$ and set at a distance $f_{D}$, so that the valve does not move since the dependency of valve output with the previous value of $\chi$ is removed. The third signal $u_{k+2}$ brings the stem position to its steady-state value $\left(\chi_{s s}\right)$ in order to eliminate error on control variable. Finally, the fourth movement $u_{k+3}$, analogously to the aim of the second move, moves towards the steady-state valve position and set the input at distance $f_{D}$, so that $\chi_{k+3}=\chi_{k+2}=\chi_{s s}$. After that, the stem cannot move from steady-state position since the input signal $u_{k+j}$ (with $j>3$ ) is always kept constant.

Note that 24) comprises actually a sequence of four moves, being based on He's semiphysical model. A simpler compensation sequence has been derived for He's standard model in [1], and indeed represents a regular two-move method, since it imposes only two different values to the valve input.

It is worth noting that the first version of two-move stiction compensation presented several drawbacks, which heavily hinder its on-line implementation [22]. Firstly, accuracy is reduced by assuming the one-parameter model of [24] to predict the valve behavior. Moreover, the steady-state value of valve position $\left(\chi_{s s}\right)$ is assumed to be known, while this variable is not usually measurable in process plants. In particular, the method relies on the strong assumption that all measurements are represented by deviation variables and their respective steady-state values are zero.

In the proposed warm-start (24), steady-state valve positions are estimated by (22), and, for transitory values of valve output $\left(\chi_{k}, \chi_{k+1}\right)$, the estimates computed along the prediction horizon $N$ are considered. This input sequence proves to be a valid warm-start for the stiction embedding MPC, by improving sig-

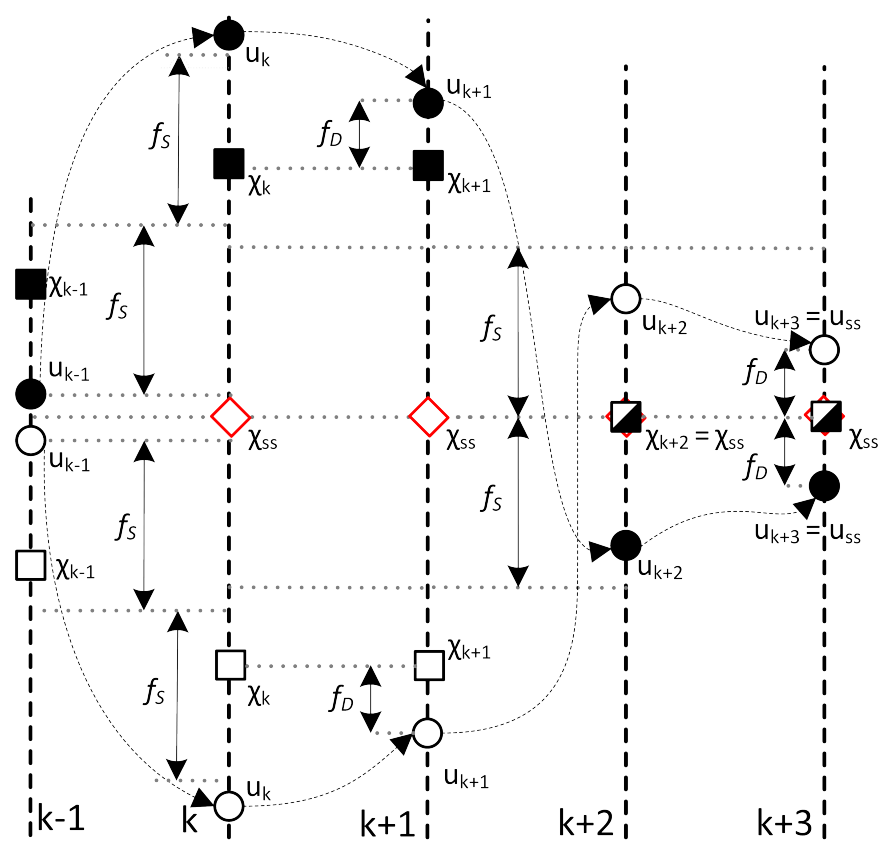

Figure 11: Sequence of moves for stiction compensation.

nificantly performance of the dynamic optimization module:

$$
\begin{aligned}
u_{1: N}^{0}= & {\left[u_{1}^{0}, u_{2}^{0}, u_{3}^{0}, u_{4}^{0}, \ldots, u_{N-1}^{0}, u_{N}^{0}\right] } \\
= & {\left[u_{0} \pm a f_{S}, \hat{\chi}_{1} \pm f_{D}, \hat{X}_{2} \pm f_{D}, \hat{\chi}_{s s} \pm f_{D}, \ldots\right.} \\
& \left.\ldots, \hat{\chi}_{s s} \pm f_{D}, \hat{\chi}_{s s} \pm f_{D}\right]
\end{aligned}
$$

where $\hat{X}_{2}=\frac{1}{M}\left[\hat{\chi}_{s s}-\hat{\chi}_{1}(1-M)\right]$.

The beneficial effect of the proposed warm-start is shown in the remainder of this section for a numerical case-study. Nominal performance is considered, since no error in process and valve dynamics is present, and no noise is added. A linear SISO system is considered for the sake of simplicity. Stiction is described by He's semiphysical model with $f_{S}=5$ and $f_{D}=2$. A third-order transfer function for the process model is considered:

$$
P(s)=\frac{1}{(10 s+1)(5 s+1)(s+1)}
$$

and the corresponding state-space model in discrete time domain with sampling period $T_{s}=1$ is obtained. Four different scenarios are analyzed:

1. standard stiction embedding MPC: no warm-start is used;

2. pure warm-start: the dynamic module of stiction embedding MPC is bypassed and the controller output corresponds exactly to the warm-start sequence;

3. stiction embedding MPC with warm-start: the sequence in (24) is given as warm-start to the controller;

4. improved stiction embedding MPC with warm-start: the proposed warm-start is used and a revised objective function is introduced.

Figure 12 shows time trends of process output and valve position of the various scenarios for the same set-point. The standard stiction embedding MPC does not move the input, thus the 

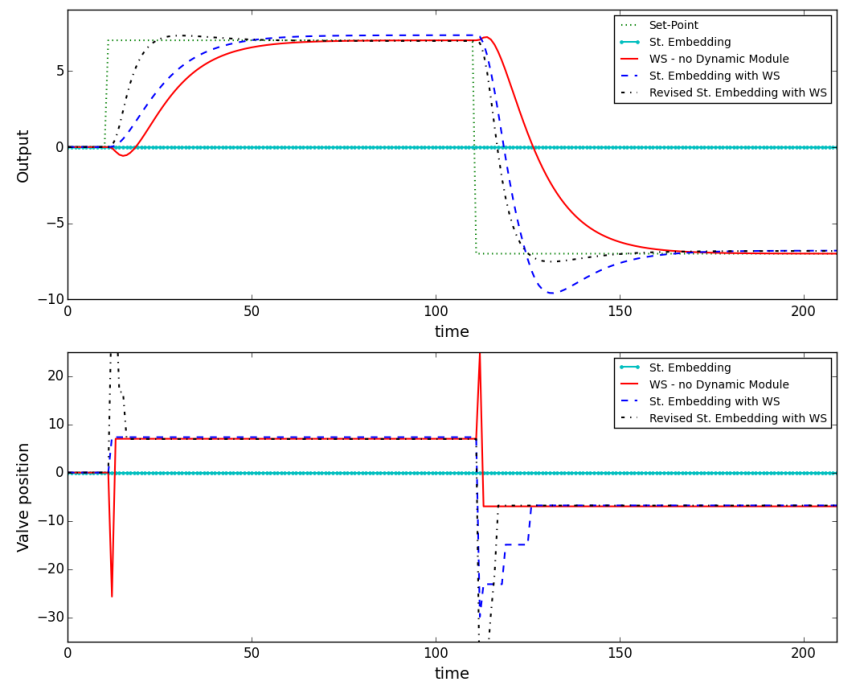

Figure 12: Beneficial of warm-start on stiction embedding MPC.

output does not reach the new set-point. The dynamic optimizer finds more convenient to stay at the initial steady-state, since, by modeling the valve deadband within the plant dynamics, the objective function could be diminished only along an unpractical prediction horizon $\left(\tilde{N} \simeq 5 \cdot 10^{4}\right.$ for this specific case). The pure warm-start excludes dynamic module of MPC and is basically open-loop mode control. This scenario allows one to get new reference with perfectly zero offset and new target for states, but with a very slow response.

The stiction embedding MPC with warm-start moves valve position when set-point changes occur, but may yield significant offsets as new targets cannot be matched always perfectly by dynamic module. Finally, in the improved stiction embedding MPC formulation, the dynamic objective function (17) is revised as follows:

$$
\ell\left(z_{i}, u_{i}\right)=\left(z_{i}-z_{s s}\right)^{T} Q\left(z_{i}-z_{s s}\right)+\Delta \hat{\chi}_{i}^{T} Q_{s} \Delta \hat{\chi}_{i}
$$

where $\Delta \hat{\chi}_{i}=\hat{\chi}_{i}-\hat{\chi}_{i-1}$ is the rate of change of the estimated valve position, and $Q_{s} \in \mathbb{R}^{m \times m}$ is the corresponding difference penalty matrix. Note that matrices $R$ and $S$ in (17) have to be set to zero accordingly, since limitations on process input are now imposed directly on the estimated valve position and not on controller output. This refined approach further reduces offset and speeds up response, but at the expense of a larger input variation and wider valve movements in transitory dynamics.

Nevertheless, it has to be remarked that the proposed warmstart has a major limitation since it is effective only for constant set-point or in the case of pure step changes. As a matter of fact, the two-move compensation which is based on is actually suitable only in the case of constant set-point, that is, when a fixed steady-state value $\chi_{s s}$ of valve position is known, measured or estimated. In the case of step changes, targets of valves position, as other states, show step variations and proposed warmstart is still effective. However, degraded performance occur when set-point is time-varying and corresponding steady-state values of valves position change along the short horizon (four moves) of the warm-start.

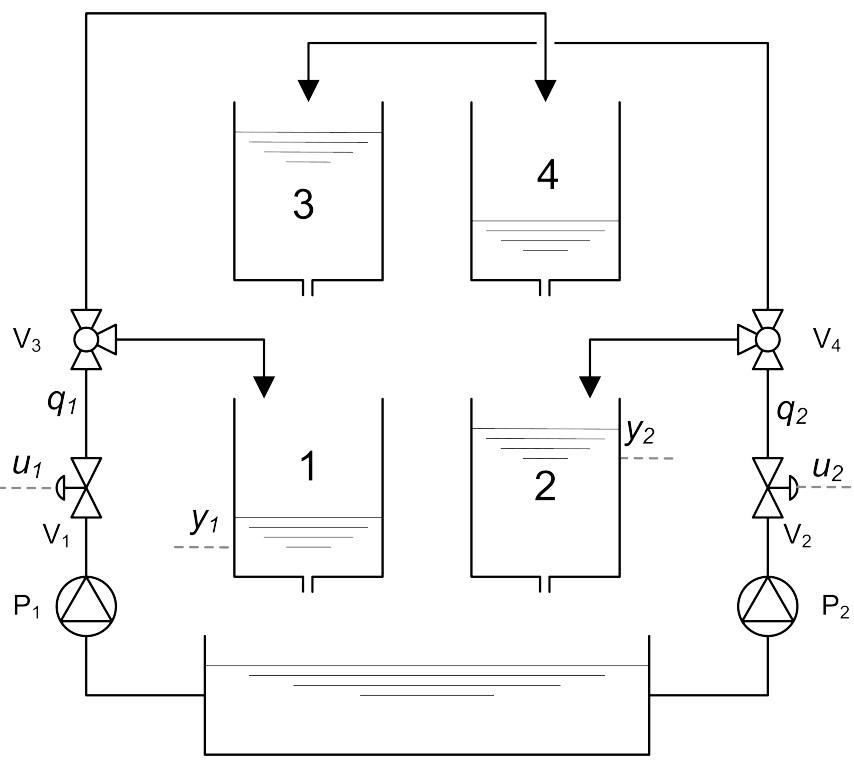

Figure 13: A multivariable nonlinear system: the quadruple-tank process.

\section{Simulation analysis}

$$
\begin{aligned}
\frac{d \xi_{1}}{d t} & =-\frac{a_{1}}{A_{1}} \sqrt{2 g \xi_{1}}+\frac{a_{3}}{A_{1}} \sqrt{2 g \xi_{3}}+\frac{\gamma_{1}}{A_{1}} K_{1} \chi_{1} \\
\frac{d \xi_{2}}{d t} & =-\frac{a_{2}}{A_{2}} \sqrt{2 g \xi_{2}}+\frac{a_{4}}{A_{2}} \sqrt{2 g \xi_{4}}+\frac{\gamma_{2}}{A_{2}} K_{2} \chi_{2} \\
\frac{d \xi_{3}}{d t} & =-\frac{a_{3}}{A_{3}} \sqrt{2 g \xi_{3}}+\frac{\left(1-\gamma_{2}\right)}{A_{3}} K_{2} \chi_{2} \\
\frac{d \xi_{4}}{d t} & =-\frac{a_{4}}{A_{4}} \sqrt{2 g \xi_{4}}+\frac{\left(1-\gamma_{1}\right)}{A_{4}} K_{1} \chi_{1}
\end{aligned}
$$

The objective of this section is to investigate the performance of the three proposed formulations of MPC in order to compensate for valve stiction. Simulations are performed on a code adapted from [25], written in Python 2.7 with the use of symbolic framework offered by CasADi 3.1. Both optimization modules of MPC implement IPOP $\mathrm{T}$ as nonlinear programming solver.

\subsection{Quadruple-tank process}

An adaptation of the well-known quadruple-tank process [26] is here considered. A schematic diagram of the system is shown in Figure 13. The target is to control the level of the lower two tanks by means of two control valves $\left(V_{1}, V_{2}\right)$. The process inputs are $u_{1}$ and $u_{2}$, that is, the output signals from MPC, and the process outputs are $y_{1}$ and $y_{2}$, level measurement of lower tanks, while level of upper tanks are assumed not measurable.

Mass balances and Bernoulli's law yield to the following continuous-time nonlinear process dynamics:

where the meaning of all variables and parameters is detailed in Table 1

The inlet flow to tank 1 is $\frac{\gamma_{1}}{A_{1}} K_{1} \chi_{1}$, and the inlet flow to tank 4 is $\frac{\left(1-\gamma_{1}\right)}{A_{4}} K_{1} \chi_{1}$. Analogously, inlet flows result for tank 2 and 
Table 1: Variables and parameters of quadruple-tank process.

\begin{tabular}{|c|c|c|}
\hline Parameter & Description [Unit] & Value \\
\hline$\xi_{i}$ & water level $[\mathrm{cm}]$ & - \\
\hline$\chi_{i}$ & valve position $[\%]$ & - \\
\hline$g$ & acceleration of gravity $\left[\mathrm{cm} / \mathrm{s}^{2}\right]$ & 981.0 \\
\hline $\mathrm{a}_{i}$ & cross-section of the outlet hole $\left[\mathrm{cm}^{2}\right]$ & $\begin{array}{l}a_{1}=0.071 \\
a_{2}=0.057 \\
a_{3}=0.071 \\
a_{4}=0.057\end{array}$ \\
\hline $\mathrm{A}_{i}$ & cross-section of tank $\left[\mathrm{cm}^{2}\right]$ & $\begin{array}{l}A_{1}=28.0 \\
A_{2}=32.0 \\
A_{3}=28.0 \\
A_{4}=32.0\end{array}$ \\
\hline$\gamma_{i}$ & flow splitting factor $\in(0,1)$ & $\begin{array}{l}\gamma_{1}=0.7 \\
\gamma_{2}=0.6\end{array}$ \\
\hline$\xi_{i}^{\max }$ & maximum tank level $[\mathrm{cm}]$ & $\begin{array}{l}\xi_{1}^{\max }=20.0 \\
\xi_{2}^{\max }=20.0\end{array}$ \\
\hline$q_{i}^{\max }$ & maximum flow rate $\left[\mathrm{cm}^{3} / \mathrm{s}\right]$ & $\begin{array}{l}q_{1}^{\max }=2 \cdot a_{1} \sqrt{2 g \xi_{1}^{\max }} \\
q_{2}^{\max }=2 \cdot a_{2} \sqrt{2 g \xi_{2}^{\max }}\end{array}$ \\
\hline$K_{i}$ & $\begin{array}{l}\text { proportional constant, valve posi- } \\
\text { tion vs. flow rate } \chi_{i}-q_{i} \text {; } \\
\text { valves with linear characteristics }\end{array}$ & $\begin{array}{l}K_{1}=q_{1}^{\max } / 100 \\
K_{2}=q_{2}^{\max } / 100\end{array}$ \\
\hline
\end{tabular}

tank 3. The modeling and control of the system have been studied at two operating points [26]. In this paper, only conditions with minimum-phase characteristics are investigated, when the control is easier and holds for $\left(\gamma_{1}, \gamma_{2}\right)>0.5$. Control valve can be subject to stiction, which is described by He's semiphysical model (5). Valve $V_{1}$ is sticky, with $f_{S}^{(1)}=6, f_{D}^{(1)}=2, M=1.99$, while valve $V_{2}$ is healthy, so that $\chi^{(2)}=u^{(2)}$. Note that stiction presence and amount are assumed known a priori for the plant model. Continuous-time dynamics of four tanks 26 is integrated using explicit Runge-Kutta 4th order method, in order to match discrete-time dynamics of two valves:

$$
\begin{aligned}
z_{k+1}=\left[\begin{array}{c}
\chi_{k} \\
\xi_{k+1}
\end{array}\right] & =\phi_{P}\left(z_{k}, u_{k}\right)=\left[\begin{array}{c}
\varphi\left(\chi_{k-1}, u_{k}\right) \\
f_{P}\left(\xi_{k}, \varphi\left(\chi_{k-1}, u_{k}\right)\right)
\end{array}\right] \\
y_{k} & =C \xi_{k}+v_{k}=\left[\begin{array}{llll}
1 & 0 & 0 & 0 \\
0 & 1 & 0 & 0
\end{array}\right] \xi_{k}+v_{k}
\end{aligned}
$$

where valves output and tanks level compose the state vector of the complete plant $z_{k}=\left[\chi_{k-1}^{T}, \xi_{k}^{T}\right]^{T}$.

\subsection{Nominal comparison}

The three proposed formulations of MPC are compared under equivalent conditions in terms of state observer and disturbance model as discussed in Section 3.2. The prediction horizon and the sampling period are set to $N=50$ and $T_{S}=5 \mathrm{~s}$. The major differences lay in the stiction compensation sequence of 24) and in the revised cost function of 25, which are respectively used within dynamic optimization module of the stiction embedding MPC formulation as warm-start and as objective function. Two different scenarios are studied:

- nonlinear models: the quadruple-tank model used within MPC formulations is nonlinear (26); only stiction unaware
MPC and stiction embedding MPC, which also implements valves nonlinearity, are derived.

- linearized model: the quadruple-tank model used within stiction inversion MPC is purely linear and the stiction nonlinearity $\varphi(\cdot)$ is inverted after optimization.

In this second scenario, the nonlinear dynamics of four tanks in 27 is linearized around the initial steady-state point $\left(\hat{\xi}_{s s}, \hat{\chi}_{s s}\right)$ :

$$
\begin{gathered}
A=D_{\hat{\xi}} f\left(\hat{\xi}_{s s}, \hat{\chi}_{s s}\right)=\left[\begin{array}{cccc}
0.9222 & 0 & 0.1958 & 0 \\
0 & 0.9451 & 0 & 0.1479 \\
0 & 0 & 0.7958 & 0 \\
0 & 0 & 0 & 0.8477
\end{array}\right], \\
B=D_{\hat{\chi}} f\left(\hat{\xi}_{s s}, \hat{\chi}_{s s}\right)=\left[\begin{array}{cc}
0.0304 & 0.0019 \\
0.0009 & 0.0231 \\
0 & 0.0162 \\
0.0110 & 0
\end{array}\right]
\end{gathered}
$$

where $D_{\hat{\xi}}(\cdot)$ and $D_{\hat{\chi}}(\cdot)$ are the Jacobians of process model dynamics with respect to tank levels $\hat{\xi}$ and valves position $\hat{\chi}$, and $\hat{\xi}_{s s}=[11.99,12.19,1.51,1.42]$, and $\hat{\chi}_{s s}=[39.58,38.15]$.

For all three formulations, tuning parameters of the static module and the terminal penalty matrix are the same: $Q_{s s}=$ $I_{2}, R_{s s}=0$, and $Q_{N}=10^{2} I_{n_{z}}$. Some differences lay in dynamic modules. In the case of stiction embedding (SE) MPC, $Q=\operatorname{diag}\left[10^{3}, 10^{3}, 1,1,10^{-6}, 10^{-6}\right], Q_{S}=\operatorname{diag}[10,100]$. For stiction unaware (SU) and stiction inversion (SI) MPC, $Q=\operatorname{diag}\left[1,1,10^{-6}, 10^{-6}\right] \simeq C^{T} C, S=\operatorname{diag}[10,100], \quad R=$ $\operatorname{diag}\left[10^{3}, 10^{3}\right]$. Note that $C^{T} C=\operatorname{diag}[1,1,0,0], Q_{(1: 2,1: 2)}^{S E}=$ $R^{S U, S I}$, and $Q_{S}^{S E}=S^{S U, S I}$, that is, tuning values are comparable among the three formulations.

Also a corresponding "ideal" MPC, with same tuning parameters, but under stiction-free environment has been considered. This nonlinear stiction-free formulation (NMPC-SF) is used as baseline for comparison, since stiction unaware MPC and stiction embedding MPC can be reduced to this controller in the absence of stiction. Figure 14 shows time trends of the tank levels, controller outputs, and valves position with different MPC formulations for the same set-points, comprised of sequences of step changes. Stiction embedding formulation can guarantee very good tracking performance with negligible offsets on both process variables, thus an effective stiction compensation is possible. Note that valve stiction is compensated so well to reproduce the behavior of the stiction unaware nonlinear controller in stiction-free environment (NMPC-SF). As a matter of fact, process outputs and valves position are substantially the same.

On the other hand, simple unaware MPC shows lower performance and does not usually remove oscillations induced by stiction, which propagate from sticky valve to all control variables. Similarly, even stiction inversion MPC, despite being aware of the valve malfunction, cannot yield good control, since the conditions (7) on input sequence to get a perfect stiction inversion, are not verified in closed-loop operation. Note that fluctuations produced by these two MPC formulations are caused by the disturbance estimate which is not zero, due to the unmodeled 
- or miss-modeled - valve dynamics. Frequencies and amplitudes of oscillation may change during the same simulation, since a dynamic state observer is used. Therefore, the effects of a time-varying gain $K_{k}(12$ on stiction induced oscillations are similar to those of a change of proportional gain $K_{c}$ in a traditional PID controller. Finally, note that controller retuning cannot completely remove these stable oscillations, but simply alters occurrences, amplitudes and frequencies.

A different version of stiction inversion MPC has also been tested. In order to fill the region where the exact inverse model $\tilde{\varphi}^{-1}$ is undefined, instead of the linear function used in $\hat{\varphi}^{-1}$ (Figure 8), a sigmoid function has been used. This modified version of stiction inversion model gives some little improvements, since lower amplitude and period fluctuations with respect to the original formulation can be obtained. However, this revised controller is not enough to give a complete stiction inversion and then to delete sustained oscillation. In the sake of space, the corresponding results are not reported.

It is also worth highlighting that the proposed fully-nonlinear formulation of stiction embedding MPC is more effective than a corresponding partly-nonlinear MPC, based on the linearized model (28), since plant-model mismatch is introduced by the linearization. A linearized stiction embedding MPC may exhibit non-negligible offsets since target calculation would be corrupted by linearization of four-tank dynamics, but detrimental oscillations would be anyway avoided. In addition, nonnegligible errors may be due to corruption of warm-start efficiency, since targets would vary also after set-point changes due to model linearization. Finally, an eventual linearized formulation of stiction unaware MPC would exhibit fluctuations of similar amplitude and frequency than corresponding nonlinear controller.

\subsection{Further results}

Some further analyses are presented in this section.

Effect of noise. The noise effect is investigated by considering all the same parameters used in nominal analysis. Eight simulations are performed with different magnitude of the output white noise covariance matrix $R_{w n}$, where $v=R_{w n}^{1 / 2} v_{r n d}$, and $v_{r n d}$ is a random sequence with normal distribution, zero-mean and unit standard deviation. The performance is evaluated using the following closed-loop objective function:

$$
\begin{aligned}
J_{C L}=\left(y-y_{s p}\right)^{T}\left(y-y_{s p}\right) & +\Delta \chi^{T} S_{p} \Delta \chi \\
& +\left(\chi-\chi^{S E}\right)^{T} R_{p}\left(\chi-\chi^{S E}\right)
\end{aligned}
$$

where $S_{p}=Q_{S}$ in the case of stiction embedding MPC, while $S_{p}=S$ for stiction unaware and stiction inversion MPC; $R_{p}=$ $Q_{(1: 2,1: 2)}$ for stiction embedding MPC and $R_{p}=R$ for other two formulations. Note that $\chi$ and $\Delta \chi$ are the value and the rate of change of actual valves position and $\chi^{S E}$ is the actual valves position for a stiction embedding MPC in nominal scenario, that is, in the case of no noise and no error on plant dynamics.

Table 2 summarizes the overall results. It can be observed that for stiction unaware and stiction inversion MPC rather constant values of $J_{C L}$ are obtained. The stiction embedding MPC produces lower values of $J_{C L}$ only until $R_{w n}=10^{-5}$, but shows a lower robustness to noise, since larger values are obtained as the noise increases with respect to other two formulations. Therefore, very good tracking performance and stiction compensation cannot be guaranteed for significant levels of noise, since non-negligible offset on controlled variables may occur.

Effect of mismatch on stiction parameters. Finally, the effect of wrong values of stiction parameters $\left(\hat{f}_{S}, \hat{f}_{D}\right)$ in the valve model of two stiction aware MPC formulations - inversion and embedding - is studied. Actual values are $f_{S}=6, f_{D}=2$, and mismatched values on static and dynamic friction are considered separately. In the first case, $\hat{f}_{S}$ is varied; in the second, $\hat{f}_{D}$ is changed. Figure 15 summarizes the whole results, by showing values of $J_{C L}(29)$ with respect to single errors: $e_{S}=f_{S}-\hat{f}_{S}$ and $e_{D}=f_{D}-\hat{f}_{D}$.

For stiction embedding MPC, as expected, minimum values of the objective function are obtained for null errors. Whereas, performance can significantly degrade when stiction parameters are wrong, underrated or overrated, as significant offsets on controlled variables may arise. Therefore, stiction embedding MPC proves to be stiction parameters dependent and is practicable only when stiction parameters are well known or in the case of slight uncertainties on valve stiction dynamics. Controller retuning could improve performance, but offsets might occur again when other operation conditions are imposed. On the other hand, stiction inversion MPC shows a higher value of performance index $J_{C L}$ in the nominal case $\left(e_{S}, e_{D}=0\right)$, but this formulation has overall a much higher robustness to errors on stiction parameters. Oscillations occur in all scenarios, only frequencies and amplitudes may change.

\section{Conclusions}

This paper has presented three different formulations of MPC to handle static friction in control valves for industrial processes. A fully unaware formulation, a stiction embedding structure, and a stiction inversion controller are designed. These model predictive controllers have been applied to multivariable processes with nonlinear systems.

It is observed that stiction embedding nonlinear MPC is the only formulation which can guarantee good performance in setpoints tracking and also stiction compensation. The two-move stiction compensation method is revised and used as warm-start to build a suitable trajectory for this MPC. In addition, some appropriate choices of objective functions and variables constraints are used with the aim of further improving performance. Nevertheless, this controller can produce non-negligible offsets when stiction is still fully modeled, but a linearization of nonlinear process dynamics is performed. In addition, a robust behavior is not possible in the presence of significant amount of white noise on the output. A similar result arises in the case of errors in the valve dynamics, that is, mismatches on stiction parameters, since offsets on process variables may be relevant.

On the other hand, the other two MPC formulations show lower compensation performance and do not completely remove oscillations induced by valve stiction in the nominal sce- 

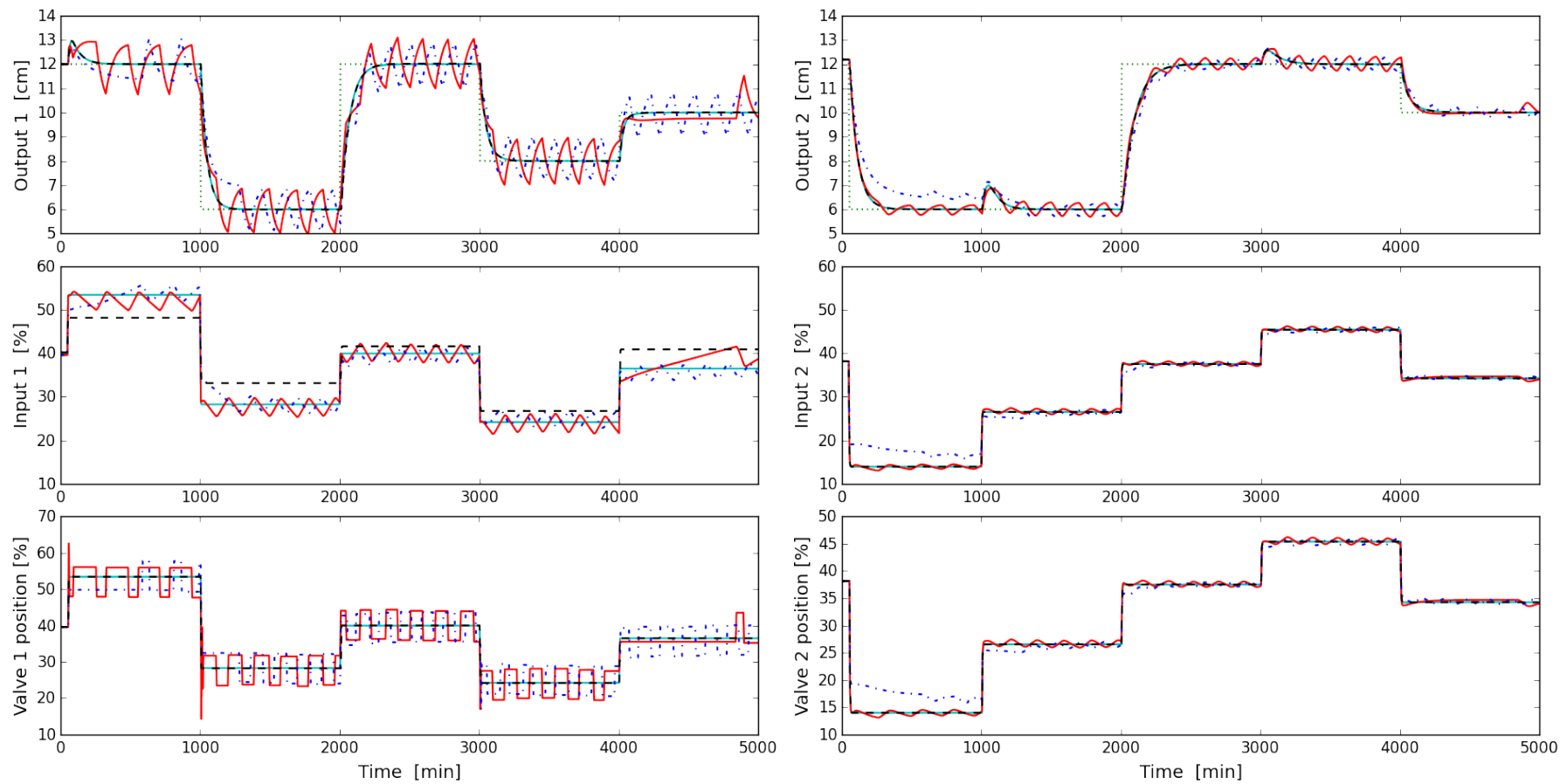

Set-Point $\quad$ NMPC-SF $\quad$ - Unaware $\quad \cdots \quad$ St. Inversion $\quad-$ - $\quad$ St. Embedding

Figure 14: Nominal comparison. Tank levels, controller outputs, and valves position for three different MPC formulations.

Table 2: Effect of noise for three MPC formulations. Values of the objective function $J_{C L}\left[\times 10^{7}\right]$.

\begin{tabular}{lcccccccc}
\hline Noise Level $\left(R_{w n}\right)$ & 0 & $10^{-9}$ & $10^{-6}$ & $10^{-5}$ & $10^{-4}$ & $10^{-3}$ & $10^{-2}$ & 0.1 \\
\hline SU-MPC & 1.6510 & 1.8130 & 1.8908 & 1.5204 & 1.6556 & 1.8591 & 1.8925 & 1.8638 \\
SI-MPC & 1.7459 & 1.2992 & 1.3418 & 1.3434 & 1.5666 & 1.4930 & 1.3238 & 1.9481 \\
SE-MPC & 0.1009 & 0.1235 & 0.8343 & 1.2361 & 4.5288 & 7.1767 & 8.7153 & 8.4120 \\
\hline
\end{tabular}

nario. Note that even stiction inversion MPC, despite being aware of the valve fault, cannot yield a good control, since conditions of discontinuity on input sequence to get perfect stiction inversion are hardly verified when this controller is implemented in closed-loop. Anyway, this formulation shows higher robustness to errors on stiction parameters, and frequencies and amplitudes of oscillation marginally change.

\section{References}

[1] R. Bacci di Capaci, M. Vaccari, G. Pannocchia, A valve stiction tolerant formulation of MPC for industrial processes, in: Proceedings of the 20th IFAC World Congress, Toulouse, France, 9-14 July, 2017, pp. 93749379.

[2] M. Jelali, B. Huang, Detection and Diagnosis of Stiction in Control Loops: State of the Art and Advanced Methods, Springer-Verlag, London, 2010.

[3] J. Tao, Y. Zhu, Q. Fan, Improved state space model predictive control design for linear systems with partial actuator failure, Ind. Eng. Chem. Res. 53 (2014) 3578-3586.

[4] A. Alanqar, H. Durand, P. D. Christofides, Fault-tolerant economic model predictive control using error-triggered online model identification, Ind. Eng. Chem. Res. 56 (2017) 5652-5667.

[5] H. Zabiri, Y. Samyudia, A hybrid formulation and design of model predictive control for systems under actuator saturation and backlash, J. of Process Contr. 16 (2006) 693-709.
[6] H. Zabiri, Y. Samyudia, MIQP-based MPC in the presence of control valve stiction, Chemical Product and Process Modeling 4 (2009) 85-97.

[7] B. Kamaruddin, H. Zabiri, Investigating MIQP-based MPC performance for stiction compensation, Australian Journal of Basic and Applied Sciences 9 (2015) 78-84.

[8] M. Rodríguez, W. Heath, MPC for plants subject to saturation and deadzone, backlash or stiction, in: Proceedings of the 4th IFAC Nonlinear Model Predictive Control Conference, Noordwijkerhout, The Netherlands, 2012, pp. 418-423.

[9] H. Durand, P. Christofides, Actuator stiction compensation via model predictive control for nonlinear processes, AIChE Journal 62 (2016) 20042023.

[10] H. Durand, P. Christofides, Empirical modeling of control valve layer with application to model predictive control-based stiction compensation, in: Proceedings of the 10th IFAC Symposium on Nonlinear Control Systems, Marriott Hotel Monterey, CA, USA, 23-25 August, 2016, pp. 4146.

[11] N. Thornhill, A. Horch, Advances and new directions in plant-wide disturbance detection and diagnosis, Contr. Eng. Pract. 15 (2007) 11961206.

[12] R. Bacci di Capaci, C. Scali, Review and comparison of techniques of analysis of valve stiction: From modeling to smart diagnosis, Chem. Eng. Res. Des. 130 (2018) 230-265.

[13] Q. P. He, J. Wang, Valve stiction modeling: First-principles vs. data-drive approaches, in: Proceedings of the 7th American Control Conference, Baltimore, MD, USA, 30 June-2 July, 2010, pp. 3777-3782.

[14] R. Bacci di Capaci, C. Scali, Process control performance evaluation in the case of frequent set-point changes with experimental applications, Can. J. Chem. Eng. 95 (2017) 1707-1720. 


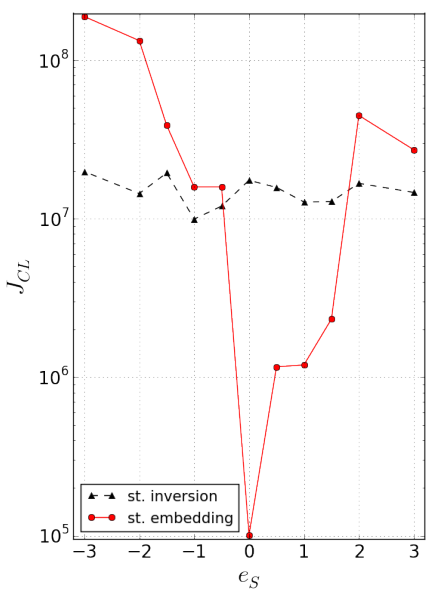

(a) errors on $f_{S}$

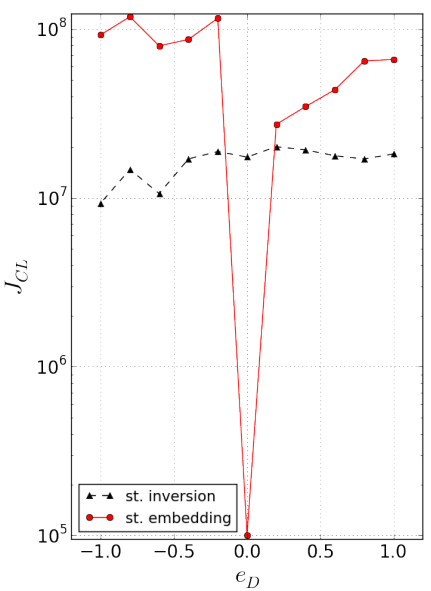

(b) on $f_{D}$.

Figure 15: Effect of mismatch on stiction parameters

[15] C. Garcia, Comparison of friction models applied to a control valve, Control Eng. Pract. 16 (2008) 1231-1243.

[16] Q. P. He, J. Wang, M. Pottmann, S. J. Qin, A curve fitting method for detecting valve stiction in oscillating control loops, Ind. Eng. Chem. Res. 46 (2007) 4549-4560.

[17] M. Kano, M. Hiroshi, H. Kugemoto, K. Shimizu, Practical model and detection algorithm for valve stiction, in: Proceedings of 7th IFAC DYCOPS, Boston, USA, 2004, paper ID n. 54.

[18] S. L. Chen, K. K. Tan, S. Huang, Two-layer binary tree data-driven model for valve stiction, Ind. Eng. Chem. Res. 47 (2008) 2842-2848.

[19] G. Pannocchia, M. Gabiccini, A. Artoni, Offset-free MPC explained: novelties, subtleties, and applications, in: Proceedings of 5th IFAC Conference on Nonlinear Model Predictive Control NMPC, Seville, Spain, 1720 September, 2015, pp. 342-351.

[20] R. Srinivasan, R. Rengaswamy, Approaches for efficient stiction compensation in process control valves, Comput. Chem. Eng. 32 (2008) 218-229.

[21] T. Wang, L. Xie, F. Tan, H. Su, A new implementation of open-loop twomove compensation method for oscillations caused by control valve stiction, in: Proceedings of 9th IFAC ADCHEM, Whistler, BC, Canada, 7-10 June, 2015, pp. 433-438.

[22] R. Bacci di Capaci, C. Scali, B. Huang, A revised technique of stiction compensation for control valves, in: Proceedings of the 11th IFAC DYCOPS, Trondheim, Norway, 6-8 June, 2016, pp. 1038-1043.

[23] L. Tang, J. Wang, Estimation of the most critical parameter for the twomovement method to compensate for oscillations caused by control valve stiction, IEEE Transaction on Control Systems Technology 24 (2016) 1869-1874.

[24] A. Stenman, F. Gustafsson, K. Forsman, A segmentation-based method for detection of stiction in control valves, Int. J. Adapt. Control 17 (2003) $625-634$.

[25] M. Vaccari, G. Pannocchia, A multipurpose, easy-to-use Model Predictive Control design and simulation code, in: Proceedings of the 4th European Conference on Computational Optimization EUCCO, Leuven, Belgium, 2016, p. 136.

[26] K. H. Johansson, The quadruple-tank process: A multivariable laboratory process with an adjustable zero, IEEE Transaction on Control Systems Technology 8 (2000) 456-465. 